\title{
Factors Affecting Use of Insecticide Treated Nets by Children Under Five Years of Age in Kenya
}

\author{
Geraldine Minoo Kyalo ${ }^{1}$, Urbanus Mutuku Kioko ${ }^{2}$ \\ ${ }^{1}$ National Treasury, Nairobi, Kenya \\ ${ }^{2}$ School of Economics, University of Nairobi, Nairobi, Kenya
}

Email address:

\section{To cite this article:}

Geraldine Minoo Kyalo, Urbanus Mutuku Kioko. Factors Affecting Use of Insecticide Treated Nets by Children Under Five Years of Age in Kenya. American Journal of Health Research. Vol. 6, No. 4, 2018, pp. 86-92. doi: 10.11648/j.ajhr.20180604.15

Received: August 26, 2018; Accepted: September 27, 2018; Published: October 25, 2018

\begin{abstract}
Malaria is a major cause of morbidity and mortality worldwide and mostly affects pregnant women and children under five years of age. It is among the leading cause of deaths in under five years of age with an approximate of 20 percent of all deaths in Kenya. The World Health Organization recommends the use of Long Lasting Insecticide Treated Nets (LLINs) and Insecticide Treated Nets (ITNs) for prevention of malaria infection. Although several studies have analysed the accessibility, availability, ownership and utilization of ITNs by children less than five years of age, information on intrahousehold factors that influence utilization of ITNs by children less than five years of age is very limited. This study investigated the factors that influence use of ITNs and LLINs by children under five years across the country using the random utility model because of the nature of the outcomes which are discrete in nature. The regression results showed that age of household head, household size, gender of child and the head of the household, presence of fever, treatment of net since acquisition significantly affected use of ITNs. The study concludes that these factors hinder use of ITNs by children under five years and therefore presence of an ITN in a household may not guarantee utilisation. The study recommends that efforts be made to increase awareness on the importance of ensuring children under five years sleep under an ITN if reduction in morbidity and mortality in this age group is to be realised.
\end{abstract}

Keywords: Malaria, Under-Five Years, Utilisation, Determinants, Binary Logit

\section{Introduction}

Malaria is a major cause of morbidity and mortality worldwide and mostly affects pregnant women and children less than five years of age [1]. World wide, close to 3.2 billion people are at risk of malaria with Sub-Saharan Africa accounting for 88 percent of malaria cases and 90 percent of malaria deaths [3]. In Kenya, malaria remains a major cause of morbidity and mortality with more than 70 percent of the population at risk of the disease [3]. It is estimated that out of a population of 46 million Kenyans, $70 \%$ are at risk of Malaria infection [2]. In Kenya, the disease accounts for 3050 percent of all hospital outpatient attendance, 19 percent of all admissions to health facilities and 3-5 percent of inpatient deaths [5].

The disease is the main cause of mortality of children under five years (approximately 20 percent) and it is ranked second after HIV/AIDs in the socioeconomic burden of diseases in Kenya $[2,19]$. In Kenya over 170 million work days are lost each year due to the disease while 4-10 million school days are lost by children [6]. The disease is not only considered as a health issue but also a socio-economic burden by the Kenyan government and therefore investment in its control should be a priority [5].

Insecticide treated nets and Long-Lasting Insecticide Nets are highly effective in reducing malaria morbidity and mortality if properly used and maintained. However, despite Kenya attaining the Roll Back Malaria (RBM) target of at 60 percent, utilization has not reached the targeted level of 80 percent. As a result, the use of ITNs remains low compared to ownership [4, 7]. In the Kenyan highlands for instance, ownership is greater than 60 percent but utilization is at 53 percent while the national average remain at only 42 percent [7]. Moreover, in 2011 only 71 percent of children below five 
years of age slept under an ITN in households which owned an ITN [5].

A number of studies have examined factors such as accessibility, availability, ownership and their effect on utilization of ITNs by children below five years of age [710]. The central question arising from these studies is whether ownership improves utilisation. In Kenya, the number of households indicating ownership of ITNs has increased over the years but utilisation has remained low. This implies that the country cannot achieve the national and international targets of ITN use unless the factors that hinder utilisation are identified and appropriate policy solutions found. In view of the foregoing, this study aimed at determining intra-household factors that may be contributing to low utilisation of ITNs by children below five years.

\section{Reviewed Literature}

Use of Insecticide treated nets is among malaria prevention measures used widely in many households. Therefore, ownership of an ITN is important in influencing the use of ITNs [11]. Due to its central role in malaria prevention, ownership of ITNs has been emphasised in many African countries $[9,12]$. For instance, ownership in Bioko Island in Equatorial Guinea was enhanced in 2007 where 110,000 LLINs were distributed to households. However, a decline of 32 percent was reported in the year 2008/09 in household ownership of ITNs and this was attributed to increase in housing and population in the country. In Sierra Leone, over 3 million Long-lasting Insecticide treated nets (LLINs) were distributed to households in an effort to protect individuals from malaria infection. This distribution increased ownership from 37 percent to 87.6 percent [12].

In areas where ownership of ITNs has been enhanced, factors such as area of residence, knowledge on malaria transmission, presence of fever in a child, age, gender and occupational status of the household head and the household size were identified as significant determinants of use of ITNs ([14]. For instance, a study in Ethiopia [14] found a unit increase in the size of household increased the odds of ownership of a net more than twice. The study further showed that households which had at least one child below five years, the odds of owning any net was about $60 \%$ higher than those with no children below five years.

In Ghana, a study on mothers' demand for preventive healthcare for children aged under-five years using a logistic regression revealed that factors such as low-income among households, age of the child, area of residence, distance to the nearest health facility and distance to nearest food market were good predictors on the mothers' adoption and utilization of ITNs [15]. The results further showed that the probability of a child to sleep under a net was inversely related with age. The findings also showed a positive relationship between low income and use of ITNs. This could be explained by the fact that households in high income bracket are in a position to afford alternative malaria prevention methods or were living in areas where mosquitoes are not a nuisance.
On the contrary [10] found a negative relationship between income and use of ITNs in Nigeria. The findings showed that children in the 2 upper quartiles were more likely to have slept under ITNs than the children in the 2 lower quartiles. These findings are supported by [7] who found a negative relationship between use of ITNs and income in Ethiopia. According to [7] factors such as gender, income of household head and presence of a radio in the household were predictors of utilization by any household member while education level of the household head and area of residence were predictors of utilization by high risk groups.

Similar findings were reported by Kenya malaria indicator survey which showed that use of ITNs decreases as the age of chid increases [5]. Although the Kenya malaria indicator survey highlights the fact that use of ITN is low at 42 percent by children below five years and that the use decreases as the age of child increases, it has not identified the intrahousehold factors that affect use of ITNS.

What is notable in these studies is that there seems to be no consensus for some of the factors which affect use of ITNs. For instance, [9] argues that use of ITNs in the rural areas is lower compared to urban areas in Equatorial Guinea while [12] claims that use is high in the rural areas than urban areas in Sierra Leone. The evidence from the literature indicates that significant effort has been made to enhance ownership in most Sub-Saharan African countries including Kenya, but utilisation of ITNs still remains below the national and regional targets compared to ownership. In view of this, it is imperative to examine factors which affect use of ITNs.

\section{Methodology}

\subsection{Theoretical Model}

In the theory of consumer choice, a consumer can either be a risk-seeker, risk-neutral or risk-averse [17, 18]. On the assumption that individual households in Kenya are riskaverse, they would choose to use an ITN or LLIN to avoid or reduce the risk of malaria infection, development of clinical disease or even death. The theory further assumes that the risk-averse behaviour emanates solely from the rational decisions of the likely outcomes and the opportunity costs of different decisions [20]. Thus, the choice problem affecting the guardian is whether to use or not to use an ITN/LLIN for children below five years of age for prevention of malaria. Further, given that children below five years of age cannot make decisions on their own the child's guardian is responsible for making decisions and the choice to take or not to take a precautionary measure is mutually exclusive.

The hypothesis is that the Childs' guardian is faced with the choice of whether to take a precautionary measure against malaria infection or not to take any precautionary measure. The discrete choice theory implies working directly with the utility functions. The choice decision of the guardian is taken to be a function of the attributes $X_{i k}$ the household $i$ perceives in the $k^{\text {th }}$ ( $k=$ use of ITN/LLIN, non-use of ITN/LLIN) choice; and households social-economic 
characteristics $\left(Y_{1}\right)$. This hypothesis can be expressed as:

$$
U_{i k}=U_{i k}\left(X_{i k}, Y_{1}\right)
$$

Where;

$U_{i k}$ is the utility that the $i^{\text {th }}$ household expects to derive from the $k^{\text {th }}$ option. The options are represented in a way that when $k=1$, it means a child below five years of age sleeps under ITN/LLIN and when $k=0$ it means a child does not sleep under an ITN/LLIN; $X_{i k}$ is a vector of use or nonuse of ITN attributes such as age and sex of guardian, knowledge on malaria treatment and intra-household factors such as family size and household characteristics while $Y_{1}$ is a vector of households socio-economic characteristics such as gender of household head.

\subsection{Econometric Model}

This study employed the random utility model because of the nature of the outcomes which are discrete in nature. The individual household's choice decision can be seen as a comparison of two rival utilities, $\bigcup_{i 1}$ and $\bigcup_{i 0}$ where $\bigcup_{i 1}$ and $\bigcup_{i 0}$ are defined as the indirect utilities, which the guardian of the $i^{\text {th }}$ individual household associates with taking a precautionary measure and not taking any precautionary measure respectively. In a linear form, the utility functions take the following form:

$$
\bigcup_{i 0}=\beta_{0}+X_{i 0} \beta_{0}+\gamma_{0} w_{0}+\varepsilon_{0}
$$

and

$$
\bigcup_{i 1}=\beta_{1}+X_{i 1} \beta_{1}+\gamma_{1} w_{1}+\varepsilon_{1}
$$

Where the observable vector of characteristics of the guardian (like age, gender) is denoted by X, while w denotes features of the two choices that are choice specific and $\varepsilon$ is the error term, which is specific to and known by the individual but not the observer.

The guardian will make a decision whether to use an ITN/LLIN based on the following options: One, the guardian will opt to use an ITN/LLIN for precautionary measure ( $k$ $=1$ ) if and only if he/she perceives there is a net welfare improvement (i.e. opt for $k^{\text {th }}$ option if $\bigcup_{i 1}>\bigcup_{i 0}$ ). Second, the guardian will choose the $k^{\text {th }}$ option if the expected benefits are equal or greater than the options opportunity cost. Assuming that the motivation of the guardian is to maximize the welfare of his/her family, he/she will choose the decision with the highest expected benefit to the family. This can be expressed as:

$$
p(k=1)=p\left(z^{\prime} \beta+\varepsilon>0\right)
$$

Where $z^{\prime} \beta$ is a collection of all the observable elements of the difference in the two utility functions and $\varepsilon$ is the difference between the two error terms. Using equation (4) above, the probability that a guardian in the $i^{\text {th }}$ household opts for a precautionary measure against malaria infection can be estimated using the following logistic regression model:

$$
p(k=1)=\frac{e^{z^{\prime} \beta}}{1+e^{z^{\prime} \beta}}
$$

Such that and $p(k=1)$ is the probability that a child sleeps under an ITN/LLIN and $z$ is a linear combination of the explanatory variables.

Linearly, this can be expressed as shown below:

$$
\begin{gathered}
\mathrm{U}=\beta 0+\beta 1 \text { agehhhead }+\beta 2 \text { agesq }+\beta 3 \text { hhsize }+ \\
\beta 4 \text { netrmratio }+\beta 5 \text { evertrted } \\
\quad \beta 6 \text { malariazon }+\beta 7 \text { sex }+\beta 8 \text { advtrtmnt }+ \\
\beta 9 \text { nonboys }+\beta 10 \text { fever } 2 \text { weeks }+ \text { ei }
\end{gathered}
$$

where; $\beta$ 's are the parameters to be estimated; Age hhhead: the age of guardian who takes care of the child, Agesq: age of the guardian squared, Hhsize: total number of persons in a household; Netrmratio: net room ratio; Ever trted: if the net was treated after it was acquired, Malaria zons: malaria zones; Sex: gender of household head; Advc trtmnt: advice on treatment; No-boys: gender of the child proxied by number of boys; Attd: attitude on malaria treatment; Fever $2 w k s$ : fever two weeks previous to the survey; $U$ : Utilization of ITNs/LLINs and $\varepsilon=$ is the error term.

In general equation (6) can be expressed as:

$$
\bigcup=\alpha+\beta X_{i k}+E_{i k}
$$

Where $U=1$ if a child under-five years of age slept under ITN/LLIN, 0 otherwise.

\subsection{Estimation Technique}

The appropriate estimation technique for equation (7) is the maximum likelihood method because Ordinary Least Squares (OLS) estimates would be biased and inconsistent due to nature of the error term which is assumed to follow a logistic distribution. Furthermore, OLS does not restrict the outcomes between 0 and 1 which is the case for binary models. We assume the existence of a binary variable $U_{i k}$ that takes the value of 1 if a child less than five years of age, $i$ sleeps under a bed net (indexed as alternative $\mathrm{k}=1$ ) and 0 otherwise (indexed as alternative $\mathrm{k}=0$ ). This can be written as:

$$
\begin{aligned}
& U_{i k}=1 \text { If } k=1 \text { (Child sleeps under a bed net) } \\
& U_{i k}=0 \text { If } k=0 \text { (otherwise) }
\end{aligned}
$$

Expressing the probability that a guardian $i$ opts for alternative 1 as $P_{i 1}=\operatorname{pr}\left(U_{i 1}=1\right)$ and alternative 0 as $P_{i 0}=1-\operatorname{pr}\left(U_{i 1}=1\right)$, then the likelihood equation can be rewritten as: 


$$
l=\prod_{u=1} \operatorname{pr}\left(U_{i 1}=1\right) \prod_{u=0} 1-\operatorname{pr}\left(U_{i 1}=1\right)
$$

Assuming further that the sample consists of independent observations on $n$ children less than five years of age, then the likelihood function is given by:

$$
l(\beta)=\prod_{u=1}^{n} F\left(z^{\prime} \beta\right) \prod_{u=0}^{n}\left[1-F\left(z^{\prime} \beta\right)\right]
$$

Where $\prod$ is the index for multiplication and indicates that the product is taken only over those cases where $u=1$ and $\mathrm{u}=0$, respectively. By taking logs, we obtain the $\log$ likelihood equation as shown in equation (11):

$$
\ln L(\beta)=\sum_{u=1}^{n} \ln F\left(z^{\prime} \beta\right)+\sum_{u=0}^{n} \ln \left[1-F\left(z^{\prime} \beta\right)\right]
$$

\subsection{Data Types and Source}

The study employed cross-sectional data from the Kenya malaria indicator survey (KMIS), a nationally representative household survey which was conducted between JulySeptember 2010. The study which was carried out by the Division of Malaria Control (DOMC) and the Kenya National Bureau of Statistics (KNBS) in collaboration with other partners was aimed at determining the status of coverage of various key malaria intervention measures like use of bed nets and coverage among other things. The KMIS data provides information on household characteristics such as age, sex; characteristics of the household dwelling unit (e.g. source of water, main cooking materials and information) on use of ITNs. A sample of 7200 households was selected to be a representative sample of the entire household population in Kenya.

After merging individual characteristics with household characteristics, the data, was transferred to STATA from SPSS. The data was then cleaned and the variables of interest were generated from the cleaned data.

\section{Findings}

\subsection{Descriptive Statistics}

Tables 1 presents descriptive statistics of the variables used in the estimation. The results showed that approximately 48 percent of the children slept under an ITN the night before the commencement of the Kenya malaria indicator survey. This is comparable to the national reported statistics which have remained relatively constant since 2007 ranging from 39.4 percent to 48.42 percent. This could be attributed to the fact that net ownership has remained almost constant within the same time period ranging between $48-50$ percent [16]. This figure is relatively low compared to the Abuja target 80 percent.

The mean age of the household head was 45 years with a maximum and minimum age of 96 and 12 years respectively.
The average total number of people living in a household varied from 1 to 28 people, with a mean value of $4.44 \pm 2.59$ per household. The net room ratio per household was $0.56 \pm$ 0.61 rooms, with a maximum of 4 nets and minimum of 0 nets.

Table 1. Descriptive Statistics (continuous variables).

\begin{tabular}{llllll}
\hline Variable & observations & mean & std dev & min & max \\
\hline $\begin{array}{l}\text { Age of house hold } \\
\text { head }\end{array}$ & 3687 & 45.33 & 16.39 & 12 & 96 \\
Age squared & 3687 & 2323.09 & 1662.12 & 144 & 9216 \\
Net-room-ratio & 3672 & .5524 & .6143 & 0 & 4 \\
No-boys & 3687 & .8207 & 1.2878 & 0 & 10 \\
Household size & 3687 & 4.4437 & 2.5923 & 1 & 28 \\
\hline
\end{tabular}

Table 2 presents a summary of descriptive characteristics of discrete variables. Only 11 percent of households treated their nets since acquisition while over 88 percent who owned a net indicated that they never treated it since acquisition. The majority $(62.7 \%)$ of the households had a female as its household head. Only 7 percent of the respondents in the sample had sought advice on malaria treatment while about 13 percent of the households had experienced fever two weeks before the Kenya malaria indicator survey.

Table 2. Descriptive Statistics (Discrete Variables).

\begin{tabular}{lll}
\hline Variable & Frequency & Percent \\
\hline $\begin{array}{l}\text { Treatment of ITNs } \\
\text { Never-treated net }\end{array}$ & 1943 & 88.52 \\
Ever-treated net & 252 & 11.48 \\
Fever 2 weeks & & \\
Presence of fever 2 weeks & 252 & 12.58 \\
No fever 2 weeks & 1973 & 87.42 \\
Advice on malaria treatment & & \\
Sought advice & 158 & 7.00 \\
Never sought advice & 2099 & 93.00 \\
Sex of household head & & \\
Male & 1377 & 37.35 \\
Female & 2310 & 62.65 \\
Malaria Zones & & \\
malaria lake & 749 & 20.33 \\
malaria high & 353 & 9.57 \\
malaria moderate & 690 & 18.71 \\
malaria seasonal & 920 & 24.95 \\
malaria low risk & 975 & 26.44 \\
Utilization & 1057 & 48.42 \\
Slept under ITN/LLIN & & 51.58 \\
Not slept under ITN/LLIN & & \\
\hline
\end{tabular}

\subsection{Econometric Results}

The results obtained using the binary logit model are shown in table 3 . The coefficients explain the changes in the probabilities of the outcome as a result of a unit change in the explanatory variables. Utilization is used as the outcome category in this equation. 
Table 3. Logit results.

\begin{tabular}{llll}
\hline Variable & coefficient & z-statistic & p-value \\
\hline age squared & .000701 & 2.58 & .010 \\
fever 2weeks & 2.051047 & 4.85 & .000 \\
advice-treatment & -.0620564 & -.11 & .914 \\
malaria low risk & -.3254693 & -1.51 & .130 \\
malaria seasonal & -.1623216 & -.78 & .437 \\
malaria moderate & -.0407963 & -.19 & .851 \\
malaria lake & -.180759 & -.89 & .371 \\
household size & .3108217 & 8.6 & .000 \\
age of household head & -.1425883 & -5.69 & .000 \\
sex & .2342167 & 1.95 & .051 \\
No-boys & .5948445 & 9.38 & .000 \\
Net-room-ratio & .0850111 & .78 & .436 \\
Ever-treated net & -.5118032 & -2.92 & .003 \\
cons & 1.96074 & 2.95 & .003 \\
no of obs=2182 & & & \\
wald chi2(15)=459.61 & & & \\
prob>chi2=0.0000 & & & \\
pseudo R2=.3409 & & & \\
log pseudolikelihood= -996.11055 & & & \\
iteration=4 & & & \\
\hline
\end{tabular}

Six variables were found to be statistically significant: age of household head, presence of fever two weeks before the Kenya malaria indicator survey, household size, sex of household, gender of the child and if the net was ever treated since acquisition. Presence of fever in a child two weeks before the national malaria indicator survey increases the log of the probability of using an ITN by 2.05 compared to those who never experienced fever two weeks prior to the survey. Presence of fever two weeks before the commencement of the Kenya malaria indicator survey is statistically significant at 0.05 level. This means that in households where a child had experienced fever before, the guardians in these households were 2.05 times more likely to make sure their children slept under an ITN as compared to those children who never experienced any fever episode before.

The age of the household head decreases the log of the probability of using an ITN by 0.14 . This means that having very old guardians was 0.14 times less likely to ensure that children slept under an ITN as compared to younger guardians. On the other hand, age squared increases the log of the probability of using an ITN by 0.0007 . This means that for the guardians who have used nets before, they are 0.0007 times more likely to make sure their children sleep under an ITN as compared to those who have never used ITNs before. Both age of household head and age squared are statistically significant at 0.05 level.

Household size increases the log of the probability of using an ITN by 0.31 and it statistically significant at 0.05 level. The average number of members in a household in the sample is four. This means that households with four or less members are 0.31 times more likely to ensure that children below five years of age sleep under an ITN as compared to those household with more than four members. Also, the gender of the household head increases the log of the probability of using ITN by 0.23 and is statistically significant at 0.05 level. This means that households headed by female heads are 0.23 times more likely to have children sleep under ITN as compared to those households headed by males.

Being a boy increased the log of probability of using ITN by 0.59 and is statistically significant at 0.05 level. This means that a boy child was 0.59 times more likely to use ITNs as compared to a girl child. The household who ever treated the net since acquisition had a negative effect on use of ITNs and this decreased the log of probability of using an ITN by 0.51 and is statistically significant at 0.05 level. This means that household which reported to have treated their nets since acquisition were 0.51 times less likely to use ITNs.

\subsection{Odds Ratio Results}

The odds ratio is the ratio between the probability of using an ITN and the probability of not using an ITN. Odds ratios are obtained by taking the exponents of the coefficients shown in table 4. The multiplicative effect implies that positive effects are greater than 1 and negative effects are between 0 and 1 .

Table 4. Odds ratio results.

\begin{tabular}{llll}
\hline Variable & odds ratio & z-statistic & p-value \\
\hline Age squared & 1.000701 & 2.58 & .010 \\
fever 2 weeks before the survey & 7.77604 & 4.85 & .000 \\
commenced & .9398299 & -0.11 & .914 \\
advice treatment & .7221883 & -1.51 & .130 \\
malaria low risk & .8501678 & -0.78 & .437 \\
malaria seasonal & .9600247 & -0.19 & .851 \\
malaria moderate & .8277237 & -0.89 & .371 \\
malaria lake & 1.364546 & 8.6 & .000 \\
Household size & .867111 & -5.69 & .000 \\
Age of household head & 1.263918 & 1.95 & .051 \\
sex & 1.812749 & 9.38 & .000 \\
no-boys & 1.088729 & 0.78 & .436 \\
net room ratio & .5994137 & -2.92 & .003 \\
ever treated net & 7.104585 & 2.95 & .003 \\
constant & & & \\
obs $=2182$ & & & \\
iteration $=4$ & & & \\
wald chi2(15)=459.61 & & & \\
prob $>$ chi2 $=0.0000$ & & & \\
pseudo $R 2=.3409$ & & & \\
log pseudolikelihood= -996.11055 & & & \\
\hline
\end{tabular}

The results reveal that six variables are statistically significant. A child having experienced fever two weeks before the commencement of the Kenya malaria indicator survey increases the ratio of probability of using ITN by 7.78 times as compared to those who never experienced fever. This implies that a child having experienced fever increases the probability of the guardian to ensure that children sleep under an ITN because they understand the dangers of malaria infection which the cost of treatment could be or even death.

The analysis further showed that families with an average of four members were 1.36 times more likely for their children below five years to sleep under an ITN compared with households with more than four household members. Sex of the household head increases the ratio of probability of using an ITN by 1.26 times implying that families whose household head was female were 1.26 times more likely to 
put a child under an ITN while sleeping than families whose household head was a male.

On the other hand, age of household head lowers the ratio of the probability of using an ITN by 0.87 times. This implies that as the household head gets more aged, the probability of using an ITN for malaria prevention reduces. On the other hand, age squared increases the ratio of the probability of using an ITN by 1.00 times. Since age squared is used as a proxy for experience, then these findings shows that as the house hold head gets more experience, the use of ITNs in the households' increases.

Low treatment of ITNS since acquisition decreases the ratio of the probability of using an ITN by 0.60 times implying that treatment of ITN influences their use negatively. This could be attributed to the fact that WHO is advocating for the use of LLINs which do not require retreatment and therefore most household could be using them. Finally, having a boy child increases the ratio of probability of using an ITN by 1.81 times. This implies that a boy child influences the use of ITNs in a household positively.

\subsection{Discussion of the Results}

The findings from this study showed that only 48 percent of children slept under an ITN. This shows a noticeable change compared to a rate of 39.4 percent reported in the Kenya malaria indicator survey in 2007 . However, this level of utilisation is still not acceptable as it falls below the 80 percent target of 2010 for children below the age of 5 years set by the African head of states in Abuja. Moreover 14 years after the RBM target declaration in Abuja, Kenya like many African countries has not met the target. In the sample, there were $51.58 \%$ children who reported not having slept under an ITN two weeks prior to the survey.

Given that some households in the sample did not own an ITN, then it is imperative for the government to ensure that more nets (and sustainable supply channels) are availed at least as urgently as the creation of awareness of the benefits of using ITNs. Awareness creation without extra campaign supply could help to increase ITN usage in areas with complementary sources of ITNs.

From the results, children who were reported to have had fever two weeks prior to the Kenya malaria indicator survey were more likely to sleep under ITNs. There was a positive association between a child having experienced fever and the use of ITNs and this was significant at $(p=0.000)$. Similar findings were reported by [9] and [10] who showed that previous episodes of fever in a child was a significant determinant of use of ITNs by children under five years.

An increased awareness of risk brought about by being sick may translate into better compliance with malaria protection. This could be explained by the consequences which come along with not using an ITN which could include hospitalization cost or even death in case of malaria infection. It is therefore important to address the misconception that children should use nets once they have been sick through information, education and communication
(IEC) messages.

Households with female heads were more likely to use ITNs with an odds ratio of 1.26 and this was significant at $(p=0.051)$. This implies that female house heads were more likely to put a child below five 5 years under an ITN during sleep than were male household heads. One of the explanations for this is that women and mothers are better in carrying out domestic chores including health-related activities; particularly child related ones such as use of ITN and administration of medicines as compared to males. It is therefore not surprising that the probability of an under-5 child to sleep under a bed net in households where a woman is the head is higher than those with males headed households. Similar findings were reported by [8] and [12] who reported female led households were more likely to use ITNs.

Number of members of a household were found to have a significant and positive effect on the use of ITNs $(p=0.000)$. This survey finds that an average of four members or less in a household influences the use of ITNs positively. Similarly, [14] found that household with four members or less were more likely to use ITNS. This could be attributed to the availability of ITNs since it is easier for few members of a household to share the ITNs as compared to when household members are many with few ITNs.

Age of household head was found to be statistically significant though it had a negative effect on use of ITNs. On the other hand, Age squared which is used as a proxy for experience affects use of ITNs positively and was statistically significant at $(p=0.01)$. This implies that age squared is an important determinant in the use of bed net by children below five years of age. Older household heads seem to use ITNs more than younger household heads. This could be associated with more exposure to awareness, education during ANC visits, and perhaps exposure to media, internet and frequent use of health facilities by the more experienced mothers. This experience potentially increases the probability of being informed about the ITN program of receiving ITNs, and the importance of using ITNs for prevention of malaria in in children below five years of age. Failure to treat ITNs affects use of ITNs negatively and is significant at $(\mathrm{p}=0.02)$.

This study found that having sought advice on malaria treatment did not have any significant effect on use of ITNs. However, previous studies such as [8] and [9] found that having knowledge on malaria transmission had a significant effect on use of ITNs.

\section{Conclusion and Policy Recommendations}

\subsection{Conclusions}

The use of ITNs has been found to be effective in preventing malaria infection. This study concludes that there are intra-household and socio-economic factors that affect utilization of ITNs within households with children below five years of age. These factors include: gender of household 
head, presence of fever in a child two weeks prior to the survey, household size gender of child, age of household head and if ITN was ever treated after acquisition. These factors hinder proper coverage of all household members and therefore presence of an ITN in a household may not guarantee utilisation.

\subsection{Policy Recommendations}

Since this study points out that expectant mothers and children under five years of age were the beneficiaries of the free distribution of ITNs, plans should be made to increase the number of free ITNs distributed to households. Such a strategy can eliminate the possibility of the adults using ITNs made for the young children who may not have the first priority for using the nets due to scarcity of ITNs in the household.

The study further found that presence of fever in a child two weeks prior to the survey had a positive impact on use of ITNs. This finding suggests the need for education as a means of achieving increased utilization of ITNs so as to avoid the misconception that children use ITNs when they are sick. Increased awareness of the risk brought by one being sick from malaria result in better compliance with malaria prevention.

The other key finding of this study which needs to be addressed is the effect of gender of both the household head and child on use of ITNs and the age of household head. Given results revealed that being a boy child increases the chances of sleeping under an ITN, it is important to highlight the importance of using ITNs for all children without discrimination by sex and all guardians and household heads, male and female, young and old should be enlightened on the benefits of using an ITN.

While recognizing that the Division of Malaria control (DOMC) cannot embark on adult education of the vast majority in the slums and rural communities where most people are either illiterate or semi-literate, it could liaise with some ministries such as the ministry of communication and education in developing and disseminating information on ITN use and benefits in the language that audience best understand.

\section{References}

[1] World Health Organization (2003). www.who.int

[2] World Health Organisation (2016). World Malaria Report

[3] Kenya Malaria Indicator Survey 2015

[4] http://www.who.int/features/2017/vector-control-kenya/en/

[5] Republic of Kenya (2009). Kenya malaria programme performance review
[6] Tilson, D (2007). The social marketing of Insecticide Treated Nets in Kenya.

[7] Atleli, H, Zhou, G, Afrane, Y, Lee, M, Mwanzo, I, Githeko, A \& Yan, G (2011). 'Insecticide treated net ownership, usage and malaria transmission in the highlands of western Kenya', Research article.

[8] Graves, P, Ngondi, J, Hwang, J, Getachew, A, Gebre, T, Wosher, A, Patterson, A, Shargle, E, Tadesse, Z, Wolkon, A, Reithinger, R, Emerson, P \& Richards, F (2011). 'Factors associated with mosquito net use by individuals in households owning nets in Ethiopia', malaria journal, vol.10 no 354.

[9] Garcia-Basteiro, A, Schwabe, C, Aragon, C, Baltazar, G, Rehman, A, Matias, A, NSeng, G \& Kleinschmidt, I (2011). 'Determinants of Bed Net Use in Children Under Five and Household Bed Net Ownership on Bioko Island, Equitorial Guinea', Malaria journal, vol.10 no.179.

[10] Oresanya, O, Hoshen, M., Sofala, O (2008). 'Utilisation of insecticide - treated nets by under - five children in Nigeria: Assessing progress towards the Abuja targets', Malaria journal vol.7 no. 145 .

[11] Hetzel, M, Gideon, G, Lero, N, Makita, L, Siba, P \& Mueller, I (2012), 'Ownership and usage of mosquito nets after four years of large scale distribution in Papua, New Guinea', Malaria Journal, vol.11 no.192.

[12] Bennett, A, Smith, S, Yambasu, S, Janbai, A, Alemu, W, Kabano, A \& Elsele, T (2012). 'Household possession and use of insecticide -treated mosquito nets in Sierra Leone 6 months after a National mass- Distribution campaign'.

[13] Astatkie, A \& Feleke, A (2009). 'Utilization of insecticide treated nets in Arbabinca Town and the malarious villages of Arbaminca Zuna District, Southern Ethiopia', Ethiopia Journal of Health development, vol.23 no.3, pp 206-215.

[14] Biadgilign, S, Reda, A \& Kedir, H (2012). 'Determinants of ownership and utilisation of insecticide-treated bed nets for malaria control in Eastern Ethiopia', Journal of Tropical medicine.

[15] Nketiah-Amponsah, E (2010). 'Mother's demand for preventive healthcare for children aged under five years: The case of utilization of insecticide treated bed nets in Ghana', Journal of sustainable development, vol.3 no.2.

[16] Kenya National Bureau of Statistics \& ICF Macro, (2011). Kenya malaria indicator

[17] Varian. H (1992). Microeconomic Analysis, Third Edition, Norton \& company, New York.

[18] Green, W (2012). Econometric Analysis, Seventh edition, Pearson education limited, London.

[19] Institute of economic affairs (2011). A cost Effectiveness Analysis: Malaria vector control in Kenya.

[20] Edward R. Moreya, Vijaya R. Sharmaa, Anne Mills (2003). Willingness to pay and determinants of choice for improved malaria treatment in rural Nepal. Social Science \& Medicine 57 (2003) 155-165 